\title{
On the independence of time and strain effects in the stress relaxation of ligaments and tendons
}

\author{
Dominique P. Pioletti ${ }^{\mathrm{a}, \mathrm{b}, *}$, Lalao R. Rakotomanana $\mathrm{a}^{\mathrm{a}, \mathrm{b}}$ \\ ${ }^{a}$ Hôpital Orthopédique de la Suisse Romande, Av. Pierre Decker 4, 1005 Lausanne, Switzerland \\ ${ }^{\mathrm{b}}$ Biomedical Engineering Laboratory, Federal Institute of Technology, Lausanne, Switzerland
}

Accepted 25 May 2000

\begin{abstract}
The hypothesis of variables separation, namely the time and the strain separation in the relaxation function, is widely used in soft tissue biomechanics. Although this hypothesis is central to several biomechanical models, only few experimental works have tried to verify it. From these studies, contradictory results have been found. Moreover, it has recently been noted that no such experimental verification has been performed for ligament tissues. In this paper, an experimental method is developed to test the hypothesis of variables separation. This method is then used with human cruciate ligaments and patellar tendons. It is shown that the use of the variables separation hypothesis is justified at least for strain values lower than $16 \%$ for anterior cruciate ligament, lower than $12 \%$ for posterior cruciate ligament and lower than $6 \%$ for patellar tendon. The method presented in this paper could be used to verify the validity of variables separation for other tissues. (C) 2000 Elsevier Science Ltd. All rights reserved.
\end{abstract}

Keywords: Stress relaxation; Variables separation; Soft tissues

\section{Introduction}

When subjected to a constant strain, the soft tissues exhibit a relaxation of the corresponding stress over time. This phenomenon is part of a general behavior called viscoelasticity. The stress relaxation has been adequately described with several models using an integral approach (Findley et al., 1976; Fung, 1993; Pioletti and Rakotomanana, 2000).

In the proposed models, the kernel of the integral - called relaxation function - depends on two variables, the time and the strain. For the identification process of the relaxation function, the hypothesis of variables separation is generally used by expressing the relaxation function in terms of two functions of one variable, the time and strain, respectively. The hypothesis of variables separation is widely used in soft tissues biomechanics (Johnson et al., 1994; Pioletti and Rakotomanana, 2000; Sauren and Rousseau, 1983; Woo et al., 1981).

\footnotetext{
* Correspondence address. Laboratoire de Genie Medical, Swiss Federal Institute Technology, PSE-Ecublens, 1015 Lausanne, Switzerland. Tel.: + 41-21-693-83-41; fax: + 41-21-693-83-30.

E-mail address: dominique.pioletti@epfl.ch (D.P. Pioletti).
}

Few experimental studies have verified the validity of the variables separation for stress relaxation tests performed on soft tissues. In some studies it has been shown that the strain levels influenced the relaxation of the stress (Haut and Little, 1972; Lanir, 1980), while in other works, only slight strain dependence has been found for the relaxation modes (Hubbard and Soutas-Little, 1984; Soden and Kershaw, 1974). The necessary experiments have not yet been done for ligament tissues (Lakes and Vanderby, 1999). As the hypothesis of variables separation is central to several models based on the integral approach, it is fundamental to perform stress relaxation tests at different strain values and to determine the correlation between the strain and the time for the relaxation process. The goal of this study is to develop an experimental method to test the hypothesis of variables separation. This method is then used to verify the validity of variables separation for human cruciate ligaments and patellar tendons.

\section{Material and methods}

Uniaxial traction tests were performed on human cruciate ligaments (anterior cruciate ligament, ACL; 
posterior cruciate ligament, PCL) and patallar tendons (PT) (5 specimens for each tissue) at controlled temperature $\left(37^{\circ} \mathrm{C}\right)$ and humidity $(100 \%)$. The specimens were successively loaded at a constant elongation rate $(0.3 \mathrm{~mm} / \mathrm{s})$ to 4 force values $(50,100,200$ and $300 \mathrm{~N})$ corresponding to strain values between $0-16 \%$ for ACL, $0-12 \%$ for PCL, and $0-6 \%$ for PT. The stress relaxation was measured for $1800 \mathrm{~s}$. To establish the reproducibility of the protocol, ligaments and tendons of calves were used first. After the last test made at the highest strain value, the specimens were reloaded to the first strain value to assess the reproducibility of the relaxation process. A complete description of the experimental part can be found in Pioletti et al. $(1996,1999)$.

A viscoelastic material undergoing uniaxial traction can be described by the relation (Findley et al., 1976; Fung, 1993; Pioletti and Rakotomanana, 2000):

$P(t)=P_{\mathrm{e}}(F)+\int_{-\infty}^{t} \kappa(F, s) \mathrm{d} s$,

where $P$ is the nominal stress, $P_{\mathrm{e}}$ the elastic nominal stress, $F$ the deformation gradient, $t$ the actual time, and $\kappa$ the relaxation function. The identification of the relaxation function $\kappa$ is performed with stress relaxation tests. However, this identification is difficult with a function of two variables. Consequently, the relaxation function $\kappa$ is generally expressed in terms of two functions of one variable, the strain and the time, respectively (hypothesis of variables separation) (Fung, 1993; Johnson et al., 1994; Pioletti, 1997; Sauren and Rousseau, 1983; Woo et al., 1981):

$\kappa(F, s)=\varphi(F) \phi(s)$.

The function $\phi(s)$ is called the reduced relaxation function. With a stress relaxation test, Eq. (2) means that the temporal behavior of the relaxation function should not be influenced by the value of the strain at which the test is performed. In case of a relaxation test performed at different deformation gradient values $F_{0}$ Eq. (1) with Eq. (2) becomes

$P(t)=P_{\mathrm{e}}\left(F_{0}\right)+\varphi\left(F_{0}\right) \int_{0}^{t} \phi(s) \mathrm{d} s$.

Determination of the integral of the reduced relaxation function is given by

$\frac{P(t)-P_{\mathrm{e}}\left(F_{0}\right)}{\varphi\left(F_{0}\right)}=\int_{0}^{t} \phi(s) \mathrm{d} s$.

We define the relative relaxation function $R\left(t ; F_{0}\right)$ as the ratio:

$R\left(t ; F_{0}\right)=\frac{\int_{0}^{t} \phi(s) \mathrm{d} s}{\int_{0}^{1800} \phi(\mathrm{s}) \mathrm{d} s}=\frac{P(t)-P_{\mathrm{e}}\left(F_{0}\right)}{P(1800)-P_{\mathrm{e}}\left(F_{0}\right)}$.
If the hypothesis of variables separation (2) is justified, the temporal behavior of the relative relaxation function $R$ should be independent of the strain $F_{0}$. This means that for tests made at different strains $F_{0}$, the value of the relative relaxation function (5) should be constant at fixed time values. Quantification of the slope of the relative relaxation over the strain values at different times will then give an indication on the validity of the hypothesis of variables separation. The more the slope percentage is close to zero (corresponding then to a horizontal curve), the more the hypothesis of variable separation is justified.

The $R(t)$ values are obtained from the stress relaxation curves. Each relaxation curve was normalized with the following relation:

\section{Normalized stress (at strain $x$ )}

$$
=\frac{\text { Stress obtained at the strain } x}{\text { Stress obtained at the highest strain }} .
$$

\section{Results}

The normalized stress relaxations for the different tissues exhibited qualitatively the same behavior (Fig. 1). A fast relaxation was observed immediately after the strain was applied, followed by a moderate relaxation until $500-1000 \mathrm{~s}$, and finally a slight relaxation till the equilibrium state (1800s).

The values of the relative relaxation function $R(t)$ for different $F_{0}$ are plotted for the same specimens (Fig. 2). The constant value for $R(t)$ reported at different initial strains means that the strain $F_{0}$ at which the stress relaxation test was performed did not influence the time behavior of the relaxation. This fact was verified for all the specimens tested and is represented in Table 1 by the low average of the slope percentage of the relative relaxation over strain values at different times.

\section{Discussion}

This paper presents an experimental verification of the hypothesis of variables separation. This hypothesis is used in several models developed for soft tissue biomechanics. The hypothesis of variables separation was experimentally validated in the present study for values lower than $16 \%$ for ACL, $12 \%$ for PCL and $6 \%$ for PT. Comparisons of slope percentages showed that the hypothesis of variables separation gives qualitatively the best results at the end of the relaxation process. The verification of the hypothesis of variables separation was important to establish as this hypothesis is widely used for the description of stress relaxation in soft tissue biomechanics. 

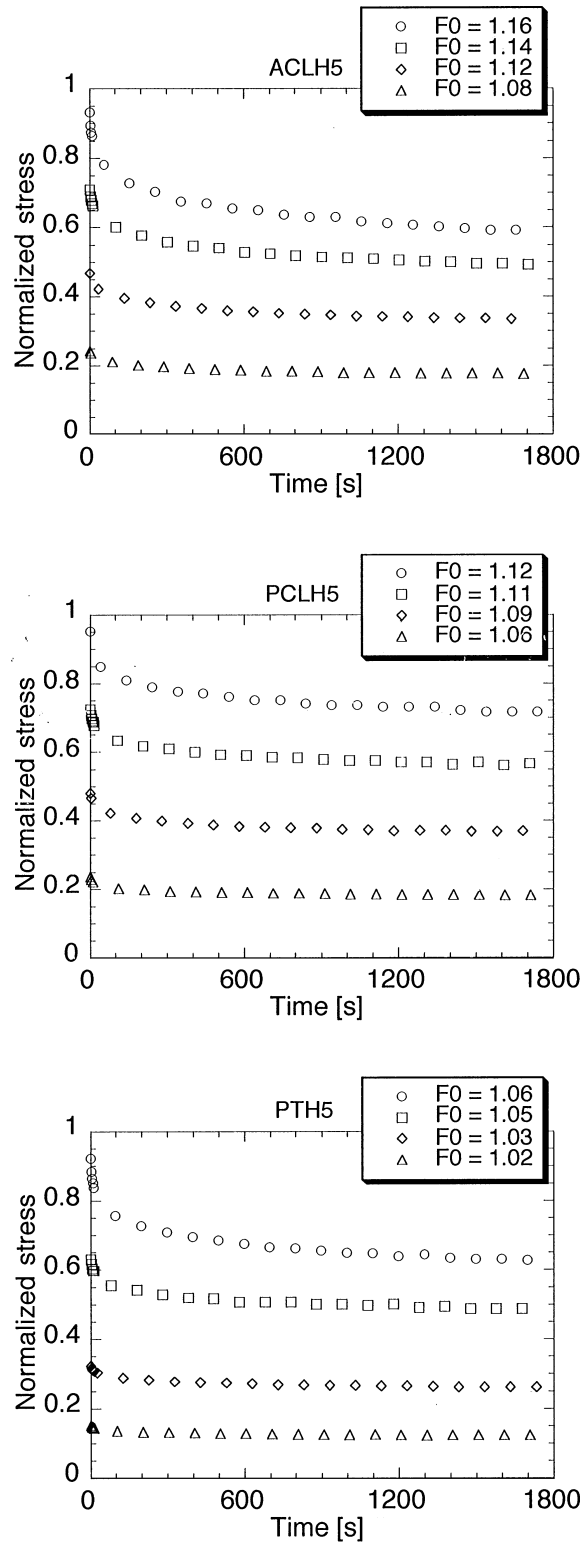

Fig. 1. Normalized stress relaxation curves at 4 strains $\left(F_{0}\right)$ for one ACL, PCL, and PT human specimen. The stress relaxation is important during the first seconds of the tests, then is moderated during the following minutes and finally reaches a quasi-static state after $1800 \mathrm{~s}$. An identical trend is found for all the specimens.

To the best of our knowledge, these results for human specimens of tendons and ligaments have not been previously reported in the literature with a quantitative method. Indeed, some previous works have checked the variables separation qualitatively from the stress relaxation curves (Haut and Little, 1972; Lanir, 1980). The present method has the advantage to quantify the strain values for which the variables separation hypothesis is valid.

The difference of the strain values used for the stress relaxation tests between specimens was due to the fact
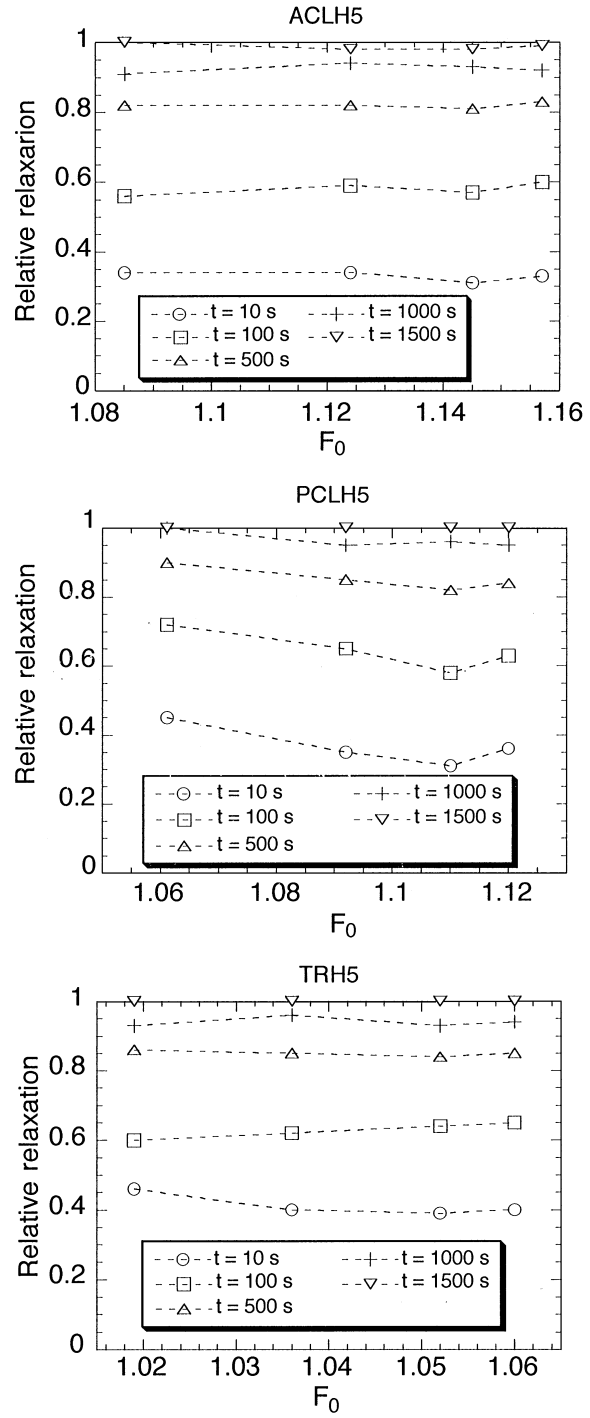

Fig. 2. Relative relaxation function $R(t)$ at different times as a function of the strain $F_{0}$. For each specimen, the value of $R(t)$ for a time value is constant at the different strain values. For the ACL specimen, the slope percentage from 1500 to $10 \mathrm{~s}$ is $0.18,0.17,0.04,0.41,0.27$, respectively. For the PCL specimen, the slope percentage from 1500 to $10 \mathrm{~s}$ is 0.01 , $0.79,1.19,1.94,1.89$, respectively. For the PT specimen, the slope percentage from 1500 to $10 \mathrm{~s}$ is $0.01,0.02,0.33,1.22,0.48$ respectively. These results enable to conclude that temporal behavior of the stress relaxation is independent of the strain value.

that we stopped the stretching process at fixed force values. As the stiffness of the specimens was different, the same force value corresponded to different elongations, then to different strain values. The control in force was chosen to preserve the mechanical integrity of the specimen between the different tests.

The use of the variables separation in integral models is then justified at least for the values tested in this study. A generalization of these results to other soft tissues seems reasonable if the composition of the tissues is mainly made of collagen as for tendons and ligaments. 
Table 1

Mean value \pm SE of the relative relaxation slope percentage for all the specimen tested at different times $(n=5)$

\begin{tabular}{rlll}
\hline & ACL & PCL & PT \\
\hline $1500 \mathrm{~s}$ & $0.6 \% \pm 0.3 \%$ & $0.4 \% \pm 0.1 \%$ & $0.5 \% \pm 0.2 \%$ \\
$1000 \mathrm{~s}$ & $0.6 \% \pm 0.3 \%$ & $1.7 \% \pm 0.6 \%$ & $1.0 \% \pm 0.6 \%$ \\
$500 \mathrm{~s}$ & $1.5 \% \pm 0.6 \%$ & $2.2 \% \pm 1.0 \%$ & $1.8 \% \pm 1.1 \%$ \\
$100 \mathrm{~s}$ & $3.9 \% \pm 2.1 \%$ & $1.9 \% \pm 0.2 \%$ & $2.9 \% \pm 1.2 \%$ \\
$10 \mathrm{~s}$ & $4.0 \% \pm 2.6 \%$ & $1.0 \% \pm 0.2 \%$ & $0.8 \% \pm 0.2 \%$ \\
\hline
\end{tabular}

Indeed, the method presented in this paper could be used to verify the validity of variables separation for other tissues.

\section{Acknowledgements}

This work was supported by the Orthopedic Hospital of Lausanne and the Biomedical Engineering Laboratory at the Swiss Federal Institute of Technology-Lausanne.

\section{References}

Findley, W.N., Lai, J.S., Onaran, K., 1976. Creep and Relaxation of Nonlinear Viscoelastic Materials. Dover Publication, New York.

Fung, Y.C., 1993. Biomechanics: Mechanical Properties of Living Tissues. Springer, New York.
Haut, R.C., Little, R.W., 1972. A constitutive equation for collagen fibers. Journal of Biomechanics 5, 423-430.

Hubbard, R.P., Soutas-Little, R.W., 1984. Mechanical properties of human tendon and their age dependence. ASME Journal of Biomechanical Engineering 106, 144-150.

Johnson, G.A., Tramaglini, D.M., Levine, R.E., Ohno, K., Choi, N.Y., Woo, S.L.Y., 1994. Tensile and viscoelastic properties of human patellar tendon. Journal of Orthopaedic Research 12, 796-803.

Lakes, R.S., Vanderby, R., 1999. Interrelation of creep and relaxation: a modeling approach for ligaments. ASME Journal of Biomechanical Engineering 121, 612-615.

Lanir, Y., 1980. A microstructure model for the rheology of mammalian tendon. ASME Journal of Biomechanical Engineering 102, 332-339.

Pioletti, D.P., 1997. Viscoelastic properties of soft tissues: application to knee ligaments and tendons. Ph.D. Thesis. EPF-Lausanne.

Pioletti, D.P., Rakotomanana, L., Gilliéron, C., Leyvraz, P.F., Benvenuti, J.F., 1996. Nonlinear viscoelasticity of the ACL: experiments and theory. In: Middletown, J. (Ed.), Computer Methods in Biomechanics and Biomedical Engineering. Gordon and Breach, London, pp. 271-280.

Pioletti, D.P., Rakotomanana, L.R., Leyvraz, P.F., 1999. Strain rate effects on the mechanical behavior of the anterior cruciate ligament-bone complex. Medical Engineering Physics 21, 95-100.

Pioletti, D.P., Rakotomanana, L.R., 2000. Non linear viscoelastic law for soft biological tissues. European Journal of Mechanics, accepted for publication.

Sauren, A.A.H.J., Rousseau, E.P.M., 1983. A concise sensitivity analysis of the quasi-linear viscoelastic model proposed by Fung. ASME Journal of Biomechanical Engineering 105, 92-95.

Soden, P.D., Kershaw, I., 1974. Tensile testing of connective tissues. Medical Biological Engineering 12, 510-518.

Woo, S.L.Y., Gomez, M.A., Akeson, W.H., 1981. The time and historydependent viscoelastic properties of the canine medial collateral ligaments. ASME Journal of Biomechanical Engineering 103, 293-298. 